\title{
PRIB-CDS: An Energy Efficient Low Duty Cycle Broadcasting Scheme for Wireless Sensor Network
}

\author{
Anubhama Ramasamy ${ }^{1 *}$ and Rajendran Thangavel ${ }^{2}$ \\ ${ }^{1}$ Department of Computer Science, Periyar University, Periyar Palkalai Nagar, Salem, 636011, Tamilnadu, India \\ ${ }^{2}$ Department of Computer Science, Government Arts and Science College, Kangeyam, Tirupur, 638701, \\ Tamilnadu, India
}

\begin{abstract}
Low duty cycling is a widely adapted technique to conserve energy in the most used Medium Access Control (MAC) protocols in Wireless Sensor Networks (WSN). But such low duty cycle-based MAC protocols perform poorly under broadcast traffic as they suffer from redundant retransmission and maximization of relay nodes problems. Addressing these issues is critical, as the advent of IoT and ubiquitous computing applications has increased the demand for broadcast support. Our previous work, Preamble based Receiver Initiated Broadcasting MAC (PRIB-MAC) performed well in most parameters under broadcast traffic, but it had scope for improvement in reducing the number of transmissions. In this paper, we propose the PRIB-Connected Dominating Set (PRIB-CDS), built on top of PRIB$\mathrm{MAC}$ with the addition of dynamic forwarding technique by forming a forwarding set with the help of Greedy algorithm. The simulation results of our proposed PRIB-CDS algorithm shows that it has reduced the number of transmissions significantly as it reduces forwarding nodes and balances the energy between the nodes to avoid re-broadcasting the data.
\end{abstract}

Keywords: Broadcasting, CDS, dynamic forwarding, MAC, PRIB-MAC, retransmission, WSN

\author{
ARTICLE INFO \\ Article history: \\ Received: 05 December 2020 \\ Accepted: 01 April 2021 \\ Published: 31 July 2021 \\ DOI: https://doi.org/10.47836/pjst.29.3.09 \\ $\overline{\text { E-mail addresses: }}$ \\ anubhama.r@gmail.com (Anubhama Ramasamy) \\ rajendran_tm@yahoo.com (Rajendran Thangavel) \\ * Corresponding author
}

\section{INTRODUCTION}

In WSN, broadcasting can be used in different scenarios like network topology discovery process, network configuration process, routing process, information dissemination and so on. In the recent developments of IoT and ubiquitous computing techniques, the sensor node can communicate via multiple hops without centralized control. Naturally, 
WSN nodes are very small in size and can function in the IoT and Ubiquitous computing applications with limited energy where the nodes can operate many years without human interruptions.

To meet the energy efficiency requirements, WSN employs various asynchronous low duty cycling techniques, where the nodes sleep and wake-up on their own schedules to conserve energy and several sender-initiated and receiver-initiated mechanisms are proposed to achieve coordination among the nodes (Anubhama \& Rajendran, 2017). But these techniques perform poorly for broadcast traffic, as the broadcasting techniques like repeated unicast proposed in Asynchronous Duty cycle Broadcasting (ADB) and flooding lead to redundancy, increased latency, and energy consumption (Sun et al., 2009; Guo et al., 2011). Therefore, providing a solution for energy efficient broadcasting in low duty cycled networks is an important issue in WSN, which we have tried to address in our previous work PRIB-MAC (Anubhama \& Rajendran, 2020b). As an alternative to repeated unicast for broadcasting, it employed short preambles as part of the receiver-initiated low duty cycle mechanism. The results were promising with improved network coverage and reduced control overhead and energy consumption.

However, re-transmission is a common issue in these low duty cycle-based protocols which can be effectively reduced by using dynamic forwarding techniques (Anubhama \& Rajendran, 2020a). In dynamic forwarding, the forwarder node is elected by using any one of the mechanisms such as Cluster based, On-demand or Multiple Criteria based, and Connected Dominating Set (CDS) based.

In Cluster based mechanism, WSN nodes form a cluster and choose the cluster head based on energy, distance, or any other criteria (Fouchal, 2012). On-demand algorithm chooses the forwarder node by calculating a threshold value, based on the residual energy level of the nodes or distance of the node from source to destination or both energy and distance. This works comparatively well in unicast than broadcast (Tan \& Chan, 2010; Rehena et al., 2013). In CDS, each node in the network must learn the 1-hop or 2-hop information of its neighbors and it chooses the forwarding node by calculating the degree of node or by using the range of each node.

Cluster based and On-demand algorithms focus more on achieving node coverage in broadcast operations (Vijayasharmila et al., 2015; Afia et al., 2019). However, these algorithms are known to have the limitation of centralized node failure and time delay, respectively. In comparison with the other dynamic forwarding algorithms, the CDS method is well employed in avoiding retransmissions and reducing the number of relay nodes, which will result in reducing energy consumption.

Based on the network topology, the CDS construction algorithms can be further classified into centralized and decentralized. In centralized, the entire control of the network is maintained by a single node and if that central control fails, the entire network collapses. In decentralized, each node manages themself, therefore a single node failure does not affect 
the entire network. The decentralized algorithm can be further classified into distributed and localized algorithms. The decision process of distributed algorithms is decentralized, and it is classified as pruning-based and MIS-based CDS construction algorithms. But the decision process of a localized algorithm is distributed where it involves continuous communication rounds and is further classified into Addition-based and subtraction-based localized algorithms. The Addition-based localized CDS construction algorithm begins with commonly disconnected nodes, and then adds other nodes to create the CDS. According to the type of initial subset, the Addition-based localized CDS construction algorithms are further classified into MIS-based algorithm and tree-based algorithm, which incorporates search techniques such as greedy and heuristic search. Subtraction-based localized CDS construction algorithms systematically remove nodes from the initial CDS, which is a set of all the nodes in the network.

The next section discusses some of the related works in forwarder selection based on distributed CDS construction algorithms. The materials and method section discusses the design and implementation of PRIB-CDS protocol, the result and discussion section describe the simulation results for PRIB-CDS in comparison with other broadcasting protocols and we conclude the paper with scope for future enhancements.

\section{RELATED WORKS}

To avoid retransmission and to achieve better broadcasting, dynamic forwarding techniques play an important role in asynchronous broadcasting MAC protocols, where election of forwarders is efficiently done using distributed CDS approach. Here some of the existing distributed CDS approaches are discussed based on their complexity, running time, stability and overhead.

The distributed pruning-based algorithm uses two phases to construct the CDS (Alzoubi et al., 2002). First, MIS (Maximal Independent Set) is identified by forming a spanning tree using the distributed leader election algorithm and next, the dominating tree is identified. All the nodes in the dominating tree form the CDS. This algorithm suffers from time and message complexity.

The Self-Pruning and Dominant-Pruning (DP) algorithms are proposed to deal with low-cost flooding tree problems by using different heuristic search flooding methods (Hyojun \& Chongkwon, 2001). In self-pruning, each node knows their 1-hop neighbour information by exchanging adjacent node information and it suffers from message overhead. Whereas in dominant pruning, each node calculates their 2-hop neighbour information by exchanging the adjacent node list with neighbour and the sender chooses the adjacent node to forward the packet to complete the flooding. But this leads to energy depletion of the source node and it also consumes more bandwidth. The improved self pruning algorithm works based on self pruning, in which every node makes its own decision to rebroadcast 
the packet depending on its neighbour details (Rab et al., 2017). It completes its broadcast with a smaller packet header, but it uses extended neighbour knowledge that contains 3-hop neighbour information, which is used to find its forwarder. This algorithm takes more computational time to decide its forwarding node. The Counter Based Dominant Pruning (CDP) algorithm is constructed by combining the advantage of counters-based scheme and Dominant Pruning (Hoque et al., 2020). It avoids the problem of the host receiving the same message again while broadcasting using some threshold value. However, this algorithm suffers from functional overheads.

In a distributed MIS-based algorithm first independent nodes are found using a searching algorithm and then MIS (Maximal Independent Set) nodes are interconnected (Das \& Bharghavan, 1997). This algorithm is further classified as single initiator and multiple initiator algorithms. Single initiators algorithm uses a MCDS (Minimum Connected Dominating Set) and spine methods to construct the routing techniques in wireless networks (Das et al., 1997). It is very expensive for maintaining. The multiinitiator algorithm proposes a message/time distributed algorithm and is designed to solve the problems in computing forwarding set by calculating MCDS (Cheng et al., 2006). But it suffers from a high message complexity problem.

The Addition based localized MIS algorithm constructs the CDS in two different stages in which Maximum Independent Set is identified by selecting the neighbor nodes with the highest count repeatedly and then applying localized searching techniques to increase additional nodes (Hong et al., 2013; Zhou et al., 2005). The tree based localized CDS algorithm is composed of three phases that are choosing the number of initiators, using the timer for forming the CDS tree, adding additional bridge nodes for connecting the neighbors respectively which results in increased time complexity.

The subtraction based localized algorithm consists of two stages to construct the CDS whereas at first each node collects and exchanges messages about its 2-hop and 1-hop neighbor details and it removes itself from the CDS if a direct connection between any pair of its 1-hop neighbors exists followed by which some heuristic search rules are applied which results in reducing the CDS size (Dai \& Wu, 2004).

Among these CDS based algorithms, the localized CDS construction algorithms work well in calculating the forwarder node and the Addition-based CDS construction algorithm produces smaller CDS than the Subtraction-based CDS construction algorithms. In comparison with the other heuristics search algorithms, greedy algorithms generate smaller CDS which in turn reduces the number of transmissions. Hence, to enhance our previous work of PRIB-MAC (Anubhama \& Rajendran, 2017) and propose an energy efficient low duty cycle broadcasting scheme for WSN, we focus on forming the CDS using a greedy algorithm. The simulation results are compared with PRIB-MAC and some of the existing state of the art broadcasting schemes like, ADB (Sun et al., 2009) and SALB (Hong et al., 2013). 


\section{MATERIALS AND METHODS}

\section{Network Model}

Let us consider there are $\mathrm{n}$ nodes in the network, which are equally distributed in equal transmission range of one unit. The given network is defined as a graph $G(V, E)$ in which $\mathrm{V}$ is the set of nodes and $\mathrm{E}$ is the edge where an edge is represented as $\{\mathrm{u}, \mathrm{v}\} \in \mathrm{E}$ where $\mathrm{u}$ and $\mathrm{v}$ are the nodes within the communication range. $\mathrm{G}$ represents a non unit disk graph. Let $\mathrm{N}(\mathrm{v})$ is a neighbour set of node $\mathrm{v}$ (including $\mathrm{v}$ ) then $\mathrm{N}(\mathrm{V})=\mathrm{Union}_{\mathrm{v} \in \mathrm{V}} \mathrm{N}(\mathrm{v})$ is a set of all nodes of $\mathrm{V}$ and $\mathrm{V}$ covers $\mathrm{U}$ if $\mathrm{U \subseteq N}(\mathrm{V})$.

A fractional $x$-hop detail of a node $v$ is a subgraph $G_{x}(v)=\left(N_{x}(v), E_{x}(v)\right)$ of the network G. Here $\mathrm{N}_{\mathrm{x}}(\mathrm{v})$ is the $\mathrm{x}$-hop neighbour set and $\mathrm{E}_{\mathrm{x}}(\mathrm{v})$ is the $\mathrm{x}$-hop connection set of $\mathrm{v}$. Now we can specify $N_{0}(v)=\{v\}$ and $N_{x}(v)=U_{u \in N x-1(v)} N(u)$ for $x \geq 1$ and $E_{x}(v)=\left\{(u, w) \mid u \in N_{x-1}(v)\right.$ $\Lambda w \in N_{x}(v)$ \} includes link among the nodes in $N_{x}(v)$. But excludes the link between two nodes which will be precisely $\mathrm{x}$ hops from $\mathrm{v} . \mathrm{G}_{\mathrm{x}}(\mathrm{v})$ collected from $\mathrm{v}$ by sending $\mathrm{x}$ rounds of "Hello" packet. It is shown in Figure 1 and if $\mathrm{v}$ has 1-hop neighbour details, then it knows all its neighbours but not the link between these neighbours. The entire $x$-hop details of a node $\mathrm{v}$ is a subgraph $\mathrm{G}_{\mathrm{x}}^{1}(\mathrm{v})=\left(\mathrm{N}_{\mathrm{x}}(\mathrm{v}), \mathrm{E}_{\mathrm{x}}^{1}(\mathrm{v})\right.$, Where $\mathrm{E}_{\mathrm{x}}^{1}(\mathrm{v})=\left\{(\mathrm{u}, \mathrm{w}) \mid \mathrm{u}, \mathrm{w} \in \mathrm{N}_{\mathrm{x}}(\mathrm{v})\right\}$ is the entire $\mathrm{x}$-hop connection set of $\mathrm{v}$ and that details is collected by sending $(\mathrm{x}+1)$ rounds of "Hello" packet. We assume each node assigns its own sleeping schedule in asynchronous manner and can transmit the data when it wakes up but receives the data only during its active time slots.

\section{Problem Formation}

In this work we consider a multisource broadcasting scheme, where every node in the network can broadcast the data to the rest of nodes in the network. Each forwarding node receives the data from source and retransmits among its neighbours to complete the broadcasting operation. Thus the broadcasting problem in WSN with different sleep/wake

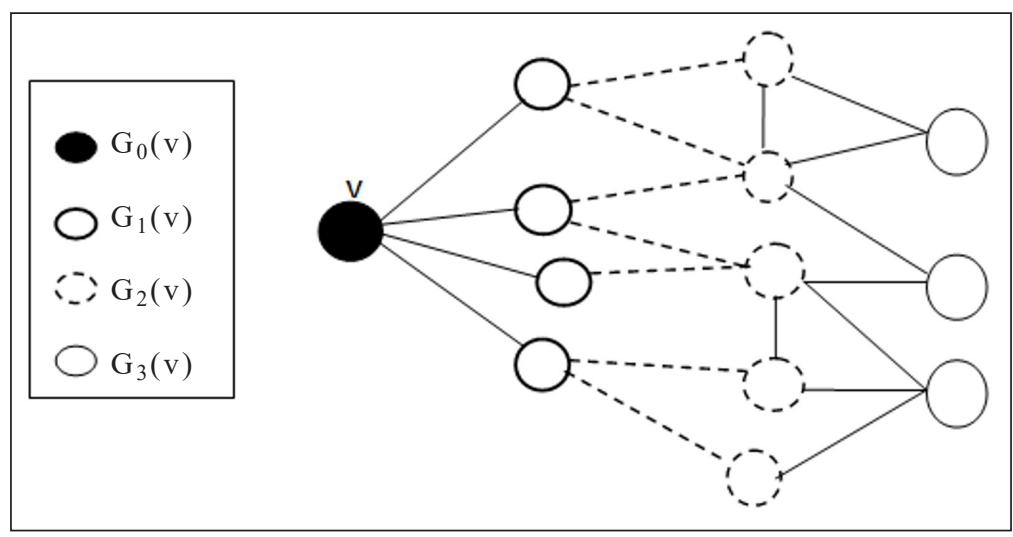

Figure 1. Fractional x-hop information 
up schedules has to be redefined by forming a forwarding set $F(G)$ from $G(V, E)$ which will be a subset of $V$. The data will be forwarded via forwarding set $F(G)$.

\section{Proposed Protocol}

PRIB-CDS is based on our existing work PRIB-MAC, in which the source broadcasts the data to the neighbour nodes by employing a short preamble. In Receiver Initiated approach, the source waits for the beacon from its neighbor nodes. Even after receiving the beacon, the source delays sending the data by sending a short preamble to its neighbors, which gives additional time for its neighbor nodes to wake up. This avoids repeated unicast by covering more nodes in a single transmission. PRIB-CDS has two key phases of operation: Construction of forwarding set and broadcast using forwarder defined in $F(G)$.

\section{Construction of Forwarding Set}

In a given network $\mathrm{G}(\mathrm{V}, \mathrm{E})$, each node knows its $\mathrm{x}$-hop subgraph $\mathrm{G}_{\mathrm{x}}(\mathrm{v})=\left(\left(\mathrm{N}_{\mathrm{x}}(\mathrm{v}), \mathrm{E}_{\mathrm{x}}(\mathrm{v})\right)\right.$ and it select the forward node set $\mathrm{F}(\mathrm{G})$ from its i-hop neighbour set $\mathrm{N}(\mathrm{v})$ to cover its next-hop neighbour set $N_{j}(v)$, that is $F(G) U\{v\}$ is a MCDS for $G_{x}(v) . F(G)$ is also known as a coverage set of $v$. When $u$ selects $v$ as a forward node then $v$ is called selector of $u$ and several selectors may exist for a single node. Statement 1 and Statement 2 define retransmission state and MCDS, respectively.

\section{Statement 1}

A forwarder will be able to transmit the message only when it receives the first packet from the selector.

\section{Statement 2}

In a given WSN network G (V,E), the nodes other than the Primary Source node (which has the data to be sent) fall under Minimum Connected Dominating Set(MCDS) for broadcasting. The forwarding set $\mathrm{F}(\mathrm{G})$ is formed from MCDS for effective broadcasting.

\section{Algorithm 1. Greedy algorithm for calculating forwarding set $F(G)$}

1: If a node is in $\mathrm{H}_{2}(\mathrm{G})$ and if it is covered only by $u$ then add $u \in \mathrm{H}_{1}(\mathrm{G})$ to $F(G)$.

2: Check the uncovered node in $F(G)$. (If a node in $\mathrm{H}_{2}(\mathrm{G})$ is not covered by $F(G)$ then it is called an uncovered node).

3: If $u$ envelops highest number of uncovered nodes in $H_{2}(G)$ then add $u \in H_{1}(G)$ to $\mathrm{F}(\mathrm{G})$.

The above greedy Algorithm 1 is used to calculate the forwarding set $F(G)$. The selection of $F(G)$ is explained in Figure 2. From that $\mathrm{N}(\mathrm{v})$ is covered when $\mathrm{v}$ transmits. 
Therefore $\mathrm{H}_{2}(\mathrm{v})=\left(\mathrm{N}_{2}(\mathrm{v})-\mathrm{N}(\mathrm{v})\right)$ is used instead of $\mathrm{N}_{2}(\mathrm{v})$. The neighbour $\mathrm{u}$ is important because a node in $\mathrm{H}_{2}(\mathrm{v})$ is only covered by $\mathrm{u}$. In the above Algorithm 1, first cover the required neighbours and then neighbours with higher degree nodes are selected to cover $\mathrm{H}_{2}(\mathrm{v})$. If two neighbours are in the same degree, any one of the nodes is selected to form a forwarding set $F(G)$. This can be identified by selecting the node with the minimum number of connected nodes.

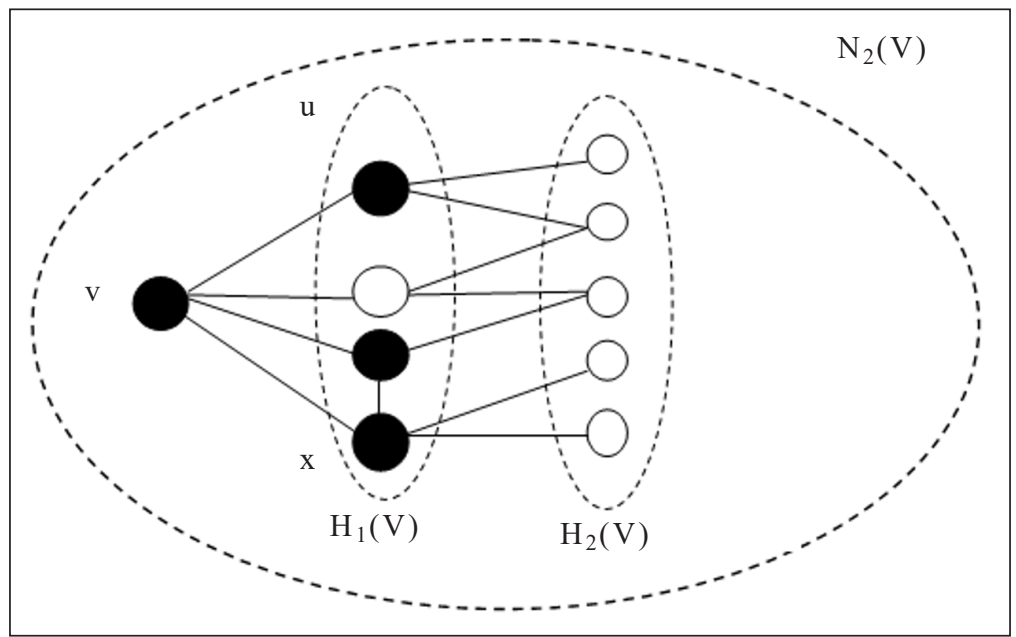

Figure 2. Each node v selects a few 1-hop neighbours to envelop its 2-hop neighbours.

\section{Broadcasting Operation in PRIB-CDS}

The Algorithm 2 presents a forwarding mechanism for the nodes in forwarding set $F(G)$ constructed by Algorithm 1.

\section{Algorithm 2. Forwarding Algorithm to Broadcast the Data}

Based on the ID and data SEQ

If the data is received for the first time then

If chanel is free and data to send then

Switch to $\mathrm{T}_{\mathrm{x}}$ mode and wait for beacon

Let FRWD_SET be the set of nodes and in F(G) except the source node $S$

If $\ni u \in$ FRWD_SET and receive beacon form the $u$ then

send a preamble tie (wait for $t_{i n}$ ) to $u$

Until $t_{\text {time out }}$ and the data broadcasting occurs.

\section{end if}

end if

end if

go to sleep mode 
In Algorithm 2, the FRWD_SET is the set of all the nodes in F(G) except the source node $\mathrm{S}$. When any of the nodes in FRWD_SET receives the beacon from its neighbour $u$, it sends a short preamble time $t_{\text {in }}$ to its receiver to keep the receiver awake until $t_{\text {time out }}$. The time $t_{\text {in }}$ is refreshed after every preamble. All the receiver nodes that wake up during the inter preamble duration send their beacons and at the end of preamble duration, the data is forwarded to all the receivers that received the preamble. Every source node maintains an ID in the broadcast packets to differentiate the data between the source nodes and adds a sequence number SEQ to identify the sequence. If every node in the forwarding set succeeds in delivering the broadcasted packets to its neighboring nodes, all nodes in the network are assured to receive the broadcast packets, as the broadcast forwarding set of PRIB-CDS is a CDS.

Minimum number of transmissions to complete a broadcast in the network is assumed to be Amin. The maximum delay time set for a source is represented as $t_{\text {time out }}$ and the number of active forwarders/receivers which would be sending the beacon to the source/ forwarders is represented as Tnodes. Also, we assume that the nodes with two extreme time differences set can be covered within two cycles of maximum delay time i.e., $2 \mathrm{t}_{\text {time out }}$.

Since the transmission to a node is only according to the number of active forwarders/ receivers in $t_{\text {time out }}$, the number of necessary transmissions is atmostmin $(\Delta, \mid$ Tnodes $\mid)$. The numbers of transmissions of PRIB-CDS are bounded by $[\min (\Delta, \mid$ Tnodes $\mid+\mathrm{c})]($ Amin + $1)$, where $\mathrm{c}$ is constant.

To cover its 2-hop neighbor nodes, the 1-hop nodes of node u need at most R2 transmission totally, where R2 is the number of node u's 2-hop neighbor nodes. Also, the 2-hop nodes of node $u$ will need to transmit messages to node u's 3-hop neighbor nodes through their 1-hop nodes, respectively. Denoting the number of node u's 3- hop neighbor nodes as R3, the numbers of transmissions to cover all 3-hop neighbor nodes of node $u$ are at most 2R3 because each 3-hop node connects to only one 1- hop node in the worst case. Therefore, the numbers of transmissions $\mathrm{M}$ to finish the broadcast equal.

$$
|\mathrm{S}|(\min (\Delta, \mid \text { Tnodes } \mid+\mathrm{R} 2+2 \mathrm{R} 3))
$$

where $\mathrm{S}$ is the dominating set. Hence a constant $\mathrm{c}$ is used to denote the upper bound of $\mathrm{R} 2+\mathrm{R} 3$.

\section{RESULT AND DISCUSSION}

The performance of PRIB-CDS is evaluated for some of the key parameters in broadcasting such as number of transmissions, network coverage and energy efficiency. The results are compared against our previous work PRIB-MAC upon which PRIB-CDS is built on and some of the state broadcasting protocols in WSN such as ADB, an RI-MAC based asynchronous duty cycled protocol and SALB, an asynchronous CDS based local broadcasting protocol for WSN. 


\section{Simulation Methodology}

The simulation is done using OMNET++ simulation engine. We deployed 6 to 21 nodes, randomly distributed over a $300 \mathrm{mX} 300 \mathrm{~m}$ square region within $125 \mathrm{~m}$ to $225 \mathrm{~m}$ transmission range where the size of each broadcast packet remains the same for every node. Preambles are special packets sent back-back to keep the node awake. Instead of sending several preambles back-back, source can send the preamble after a particular interval of time, which is less than the data time out interval of receivers. The parameters used in the simulation environment are given in Table1.

Table 1

Parameters used in simulation

\begin{tabular}{ll}
\hline \multicolumn{1}{c}{ Parameter } & \multicolumn{1}{c}{ Value } \\
\hline Area & $300 \mathrm{mX} 300 \mathrm{~m}$ \\
Total No.of nodes & 6 to 21 \\
Transmission range & $125 \mathrm{mX} 225 \mathrm{~m}$ \\
Time Slot & $1 \mathrm{~s}$ \\
Time interval per hop & $0.3 \mathrm{~s}$ \\
Preamble Duration & $0.002 \mathrm{~s}$ to $0.05 \mathrm{~s}$ \\
\hline
\end{tabular}

\section{Simulation Results}

Number of Transmissions. The line chart in Figure 3 shows the number of transmissions in PRIB-CDS and PRIB-MAC with varying preamble durations. For PRIB-MAC, it takes 8 transmissions for $100 \%$ coverage in a 7 -node network and the preamble duration has no effect on the number of transmissions as it suffers from retransmission issues. Use of dynamic forwarding in PRIB-CDS shows significant improvement in reducing the number of transmissions as the preamble duration increases.

Figure 4 compares the number of transmissions in PRIB-CDS, ADB, PRIB-MAC and SALB for different network sizes. In ADB, the number of transmissions increases exponentially for a 21-node network to 43 as it performs repeated unicast transmission to achieve broadcasting. Similarly, PRIB-MAC shows linear increase of 25 transmissions for 21 node networks as it suffers from retransmission issues. SALB performs better as the

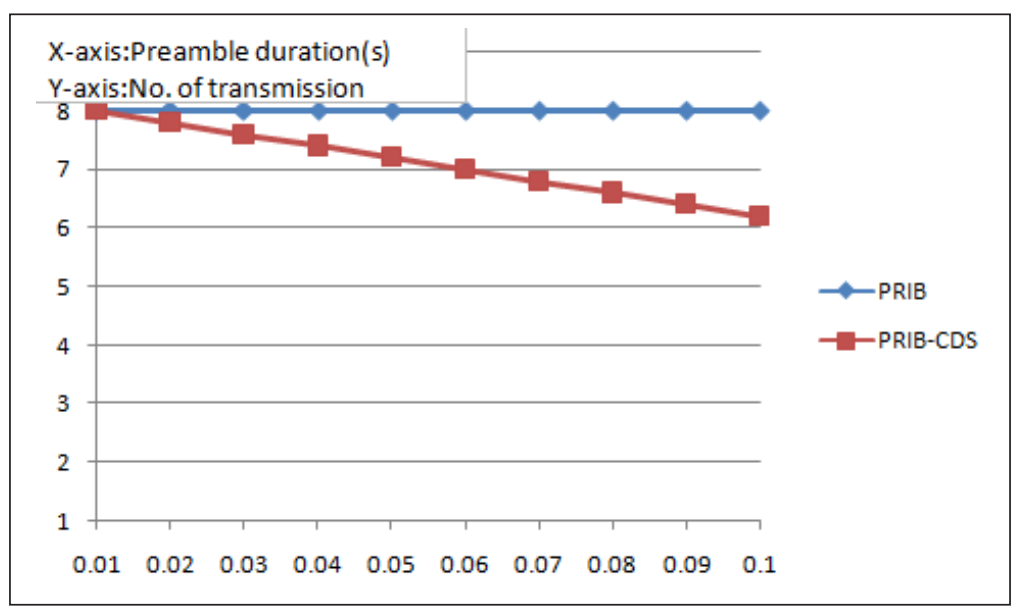

Figure 3. Number of transmissions with different preamble duration 


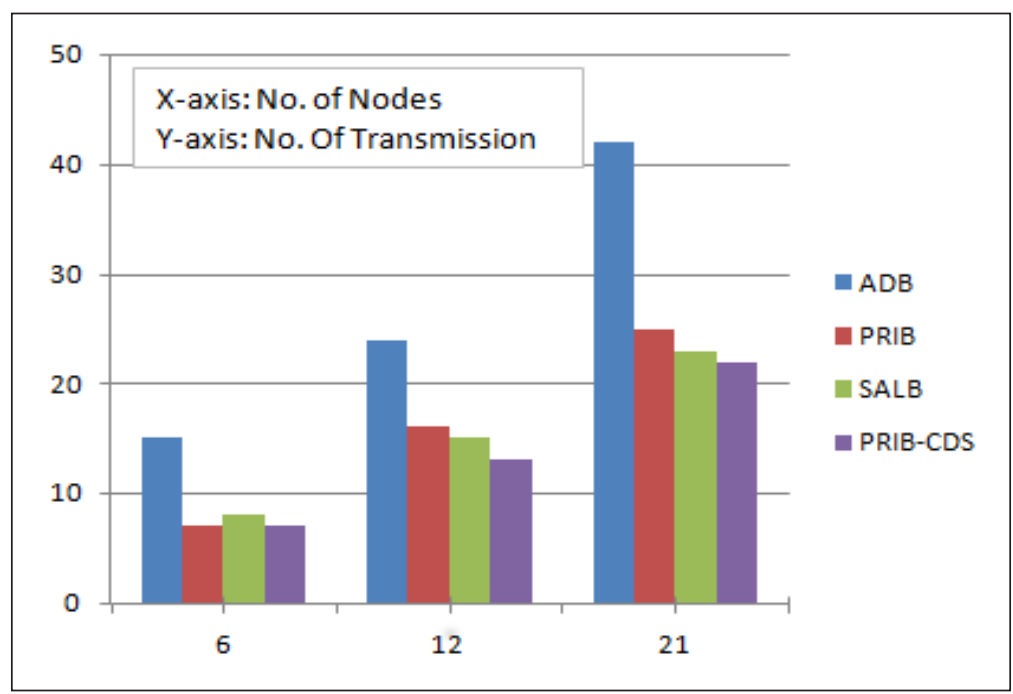

Figure 4. Number of transmissions for different network sizes

network size scales up i.e, 22 transmissions for 21 node networks but when the network size is small it needs 9 transmissions for 6 node networks as each dominator must transmit to its dominant one by one, and there exists redundant paths between the dominators in the forwarding set. PRIB-CDS shows marked improvement across network sizes due to the combination preamble-based approach and the CDS based dynamic forwarding technique.

\section{Network Coverage Time}

The line chart in Figure 5 shows the network coverage of PRIB-CDS compared against SALB, PRIB-MAC and ADB. PRIB-CDS achieves full coverage within 3.05s compared with ADB, PRIB-MAC. ADB performs poorly as it requires repeated transmission to

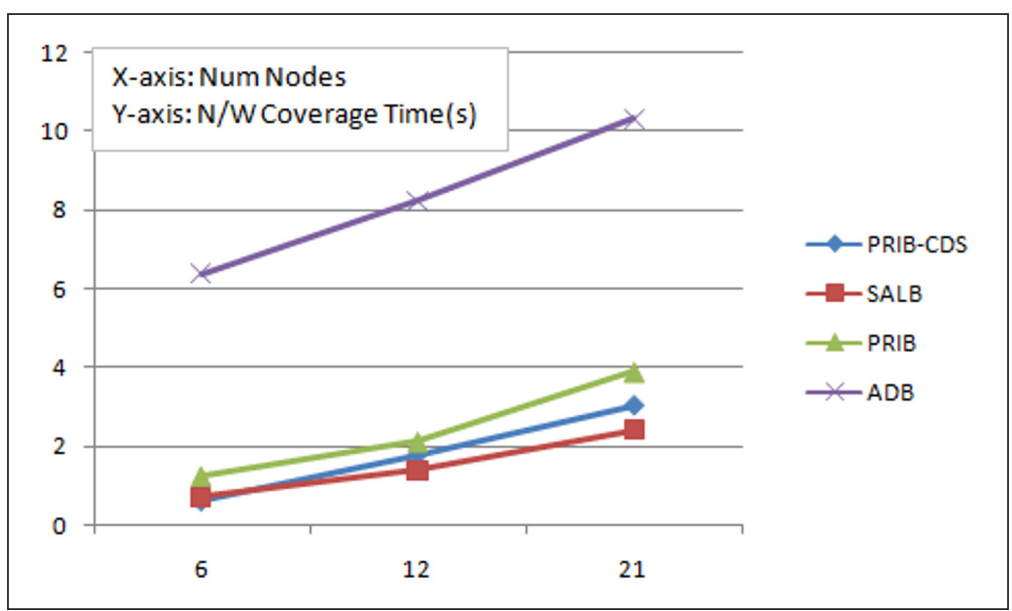

Figure 5. Network coverage time(s) with different numbers of nodes 
achieve full coverage. All the other three protocols perform reasonably well with PRIBMAC taking slightly more time due to preamble delays and retransmissions issues due to the lack of proper forwarding techniques. Both PRIB-CDS and SALB perform well with SALB doing slightly better than PRIB-CDS. It must be noted PRIB-CDS takes a slightly more time than SALB $(2.5 \mathrm{~s})$ though it performs better in number of transmissions. It could be caused by delay due to contention in PRIB-CDS. In Figure 2 shows that two adjacent nodes could be selected as forwarder nodes even if they lie within the transmission range of one another and when these nodes try to rebroadcast the data, they will access the medium at the same time resulting in contention.

\section{Energy Consumption}

Figure 6 shows the average energy consumption in percentage for PRIB-CDS and other protocols. As expected, ADB consumes more energy than the other protocols because of its repeated unicasting approach. To keep the receiver active, senders send lots of beacons which results in high energy consumption (34\% to 36\%) for both sender and receiver nodes as the repeated unicast approach results in redundant transmissions. PRIB-MAC performs poorly (11\%) compared to PRIB-CDS (4\%) as it suffers from retransmission issues during broadcasting due to lack of a proper forwarding mechanism. The line chart shows that PRIB-CDS performs the best with 4\% consumption for 21 node networks and the average energy consumption decreases as the network size increases. It is slightly better than SALB (5\%) as the sender nodes employ short preambles to cover more nodes in the asynchronous duty cycle. Furthermore, in SALB, when the size of the network is small, each dominator transmits to its dominant one by one, which is the same as repeated unicast resulting in higher energy consumption.

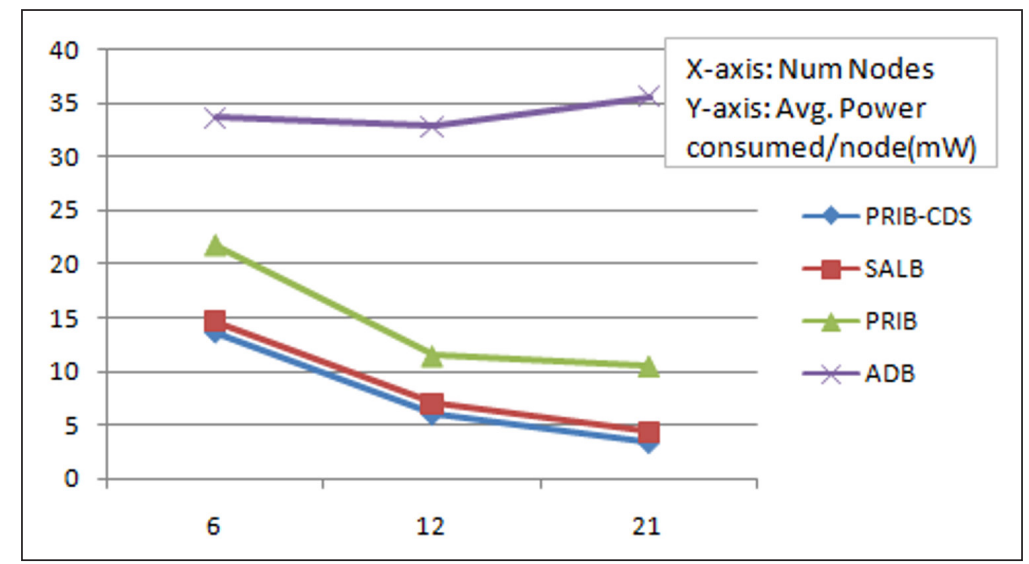

Figure 6. Average energy Consumption (\%) 


\section{CONCLUSION}

We have presented an efficient and robust broadcasting scheme PRIB-CDS, for low duty cycled Wireless Sensor Networks, which builds on to the advantages of the preamblebased approach in PRIB-MAC, by reducing the number of retransmissions by forming a forwarding set. Our proposed PRIB-CDS algorithm uses a CDS based approach to find the forwarder node in addition to the use of short preambles, which improves key performance metrics of broadcasting such the number of transmissions, average energy consumption and network coverage time when compared to the multiple unicast approach proposed in ADB. Comparisons against another CDS based approach in SALB shows significant reduction in the number of transmissions and energy consumption, with scope for improvement in delay to achieve $100 \%$ node coverage.

\section{ACKNOWLEDGEMENT}

The author would like to thank her brother and sister for providing support to this research work and grateful to peer reviewers who have saved her from many mistakes through comments. The author hopes this research can provide benefits and contribute to further research.

\section{REFERENCES}

Afia A., Tahrima O., Diana S., \& Ferdous, C. N. (2019). Construction of connected dominating set to reduce contention in wireless ad-hoc network. In 6th International Conference on Networking Systems and Security (pp. 59-67). Association for Computing Machinery. https://doi.org/10.1145/3362966.3362975

Alzoubi, K. M., Wan, P. J., \& Frieder, O. (2002). New distributed algorithm for connected dominating set in wireless ad hoc networks. In Proceedings of the 35th Annual Hawaii International Conference on System Sciences (pp. 3849-3855). IEEE Conference Publication. https://doi.org/10.1109/HICSS.2002.994519

Anubhama, R., \& Rajendran, T. (2017). A survey on MAC protocols for wireless sensor networks. Annals of Computer Science and Information Systems, 10, 121-126. http://dx.doi.org/10.15439/2017R26

Anubhama, R., \& Rajendran, T. (2020a). A study of dynamic forwarding techniques in wireless sensor networks. In M. Sundaresan \& W. R. Varuna (Eds.), Proceeding of International E-Conference of Innovations in Information Technology (IECIIT-2020) (pp. 28-34). Coimbatore Institute of Information Technology, India.

Anubhama, R., \& Rajendran, T. (2020b). PRIB-MAC: A preamble-based receiver initiated MAC protocol for broadcast in wireless sensor networks. Sādhanā, 45(1), Article 79. https://doi.org/10.1007/s12046$020-1322-7$

Cheng, X., Ding, M., Du, D. H., \& Jia, X. (2006). Virtual backbone construction in multihop ad hoc wireless networks. Wireless Communications and Mobile Computing, 6(2), 183-190. https://doi.org/10.1002/ wcm. 378 
Dai, F., \& Wu, J. (2004). An extended localized algorithm for connected dominating set formation in ad hoc wireless networks. IEEE Transaction on Parallel Distributed Systems, 15(10), 908-920. https://doi. org/10.1109/TPDS.2004.48

Das, B., \& Bharghavan, V. (1997). Routing in ad-hoc networks using minimum connected dominating sets. In Proceedings of ICC'97-International Conference on Communications (Vol. 1, pp. 376-380). IEEE Conference Publication. https://doi.org/10.1109/ICC.1997.605303

Das, B., Sivakumar, R., \& Bharghavan, V. (1997). Routing in ad hoc networks using a spine. In Proceedings of Sixth International Conference on Computer Communications and Networks (pp. 34-39). IEEE Conference Publication. https://doi.org/10.1109/ICCCN.1997.623288

Fouchal, S., Monnet, Q., Mansouri, D., Mokdad, L., \& Ioualalen, M. (2012). A clustering method for wireless sensors networks. In 2012 IEEE Symposium on Computers and Communications (ISCC) (pp. 000888000892). IEEE Conference Publication. https://doi.org/10.1109/ISCC.2012.6249414

Guo, S., Kim, S. M., Zhu, T., Gu, Y., \& He, T. (2011). Correlated flooding in low-duty-cycle wireless sensor networks. In 2011 19th IEEE International Conference on Network Protocols (pp. 383-392). IEEE Conference Publication. https://doi.org/10.1109/ICNP.2011.6089078

Hyojun, L., \& Chongkwon, K. (2001). Flooding in wireless ad hoc networks. Computer Communications, 24(3-4), 353-363. https://doi.org/10.1016/S0140-3664(00)00233-4

Hong, J., Li, Z., Lu, D., \& Lu, S. (2013). Sleeping schedule-aware local broadcast in wireless sensor networks. International Journal of Distributed Sensor Networks, 9(12), 451970. https://doi.org/10.1155/2013/451970

Hoque, S., Majumder, R., Islam, S., \& Anannya, T. T. (2020). Reducing redundancy by optimizing dominant pruning algorithm for wireless ad hoc networks. In Proceedings of the International Conference on Computing Advancements (pp. 1-9). Association for Computing Machinery. https://doi. org/10.1145/3377049.3377073

Rab, R., Sagar, S. A. D., Sakib, N., Haque, A., Islam, M., \& Rahman, A. (2017). Improved self-pruning for broadcasting in ad hoc wireless networks. Wireless Sensor Network, 9(02), 73-86. https://doi.org/10.4236/ wsn.2017.92004

Rehena, Z., Roy, S., \& Mukherjee, N. (2013). Efficient data forwarding techniques in Wireless Sensor Networks. In 2013 3rd IEEE International Advance Computing Conference (IACC) (pp. 449-457). IEEE Conference Publication. https://doi.org/10.1109/IAdCC.2013.6514268

Sun, Y., Gurewitz, O., Du, S., Tang, L., \& Johnson, D. B. (2009). ADB: An efficient multihop broadcast protocol based on asynchronous duty-cycling in wireless sensor networks. In Proceedings of the 7th ACM conference on embedded networked sensor systems (pp. 43-56). Association for Computing Machinery. https://doi.org/10.1145/1644038.1644044

Tan, H. X., \& Chan, M. C. (2010). A²-MAC: An adaptive anycast mac protocol for wireless sensor networks. In 2010 IEEE Wireless Communication and Networking Conference (pp. 1-6). IEEE Conference Publication. https://doi.org/10.1109/WCNC.2010.5506098

Vijayasharmila, S., Kumar, P. G., \& Kamalesh, S. (2015). A survey on connected dominating sets (CDS) both in the wireless sensor networks and wireless ad hoc networks. International Journal of Engineering Research \& Technology, 4(2), 898-905. 
Zhou, D., Sun, M. T., \& Lai, T. H. (2005). A timer-based protocol for connected dominating set construction in IEEE 802.11 wireless networks. In The 2005 Symposium on Applications and the Internet (pp. 2-8). IEEE Conference Publication. https://doi.org/10.1109/SAINT.2005.9 